\section{CD-ROM zur Asthmatherapie}

Mit der CD-ROM „Hintergrundinformationen zu Inuvair ${ }^{\odot}$ Mikrosol“ bietet Janssen-Cilag einen Service mit wichtigen Informationen rund um die Asthmatherapie mit der innovativen Fixkombination. Sie enthält einen Powerpointvortrag mit rund 60 Folien, in dem praxisrelevante und wissenschaftliche Informationen, von der Anwendung bis zu einem umfassenden Studienüberblick, interessant und ansprechend aufbereitet sind. In Animationen, Grafiken und Abbildungen wird über die Wirkung von Kombinationspräparaten auf Symptome und Entzündung bei Asthma genauso informiert, wie über Unterschiede von Inhalationssystemen und die Bedeutung der Partikelgröße für die Lungendeposition in den kleinen Atemwege. Der Vortrag eignet sich für die individuelle Fortbildung am PC, kann aber auch für eigene Vortragsveranstaltungen eingesetzt werden. Interessierte können die kostenlose CD-ROM unter der E-Mail: janssencilag@medizin.pr.de bestellen.

Pressemeldung Janssen-Cilag, 8.07.2010

\section{Progressionsfreies Überleben bei NSCLC verdoppelt}

Die Erstlinientherapie des fortgeschrittenen nicht kleinzelligen Lungenkarzinoms (NSCLC) mit Gefitinib (IRESSA) verbessert signifikant das progressionsfreie Überleben. In einer prospektiven, randomisierten Phase-III-Studie wurde Gefitinib mit einer platinhaltigen Kombinationschemotherapie, dem derzeitigen Behandlungsstandard, verglichen (Maemondo $M$ et al. N Engl J Med 2010;362: 2380-8). Die Studie, an der nur Patienten mit aktivierender EGRF-Mutation teilnahmen, erreichte den primären Endpunkt mit einer Verdopplung des progressionsfreien Überlebens (10,8 vs. 5,4 Monate). Gegenüber dem Carboplatin/PaclitaxelRegime senkte Gefitinib das Progressionsrisiko um $70 \%$ (HR = 0,30, 95\% KI $0,22-0,41, p<0,001)$. Auch bei dem sekundären Endpunkt, dem Gesamtüberleben, zeigte sich ein Trend zu Gunsten des IRESSA-Regimes, unter dem die Patienten fast sieben Monate länger überlebten (30,5 vs. 23,6 Monate, $p=0,31$ ). Da Patienten mit positivem EGFR-Mutationsstatus besonders profitieren, steht mit IRESSA eine so genannte personalisierte Therapie zur Verfügung.

\title{
PNEUMONEWS
}

\section{Herausgeber Klinik:}

Prof. Dr. A. Gillissen, Kassel

Prof. Dr. M. J. Kohlhäufl, Stuttgart-

Gerlingen

\section{Schriftleiter:}

Priv.-Doz. Dr. S. Budweiser, Rosenheim (Beatmungsmedizin)

Prof. Dr. G. Friedel, Stuttgart-Gerlingen (Thoraxchirurgie)

Prof. Dr. A. Ghofrani, Gießen, Dr. K. Gutjahr, Leipzig (Erkrankungen des Lungenkreislaufs)

Prof. Dr. A. Gillissen, Kassel (Asthma bronchiale)

Prof. Dr. M. Griese, München (Pädiatrische Pneumologie)

Prof. Dr. A. Günther, Prof. Dr. Ph. Markart, Gießen (Interstitielle Lungenerkrankungen)

Prof. Dr. M. J. Kohlhäufl, Stuttgart-Gerlingen (Tumoren)

Dr. S. R. Ott, Bern (Infektiologie)

Prof. Dr. K. Rasche, Wuppertal, Prof. Dr. K.-H. Rühle, Hagen, Prof. Dr. H. Schäfer, Völklingen (Schlafmedizin)

Prof. Dr. G. Rohde, Maastricht/Niederlande (COPD, Emphysem)

Dr. K. Schultz, Bad Reichenhall (Rehabilitation)

Prof. Dr. M. Schwab, Prof. Dr. K. Mörike, Tübingen (Pharmakologische Therapie)

\section{Redaktion:}

Dr. Beate Schumacher (Chefredaktion, verantwortlich, Anschrift wie Verlag, beate.schumacher@springer.com) Ute Kempf (ute.kempf@springer.com),Dr.Judith Neumaier (judith.neumaier@t-online.de), Dr. Thomas Riedel, Angelika Holzgreve (Assistenz Chefredaktion, angelika.holzgreve@ springer.com)

\section{Verlag:}

Springer Medizin, $\odot$ Urban \& Vogel $\mathrm{GmbH}$, Aschauer Str. 30, D-81549 München, Tel.: o 89/20 30 43-13 oo, Fax: -13 99, http://www.urban-vogel.de. Springer Medizin ist Teil der Fachverlagsgruppe Springer Science+Business Media.

\section{Geschäftsführer:}

Harm van Maanen, Dr. med. Dirk Einecke, Ulrich Huber, Stephan Kröck, , Dr. med. Esther Wieland, Matthias Wissel.

\section{Anzeigenverkauf:}

Ines Spankau (Anzeigenleitung, -1339, verantwortlich, Anschrift wie Verlag), Anzeigenpreisliste Nr. 4 vom 1. Oktober 2010.

\section{Corporate Publishing:}

Dr. Ulrike Fortmüller (Leitung München, -1417)

\section{Herstellung:}

Johannes Buchmann (Leitung); w\&Co MediaServices München $\mathrm{GmbH} \&$ Co KG (Layout); Dieter L. Adam, München (Titelbild)

\section{Vertrieb/Marketing Services:}

Frank Niemann (Leitung, -14 11)

\section{Abonnement:}

Die Zeitschrift erscheint 6 x jährlich. Neue Bezugspreise ab 2011: Einzelheft $12 €$ inkl. der deutschen MwSt. zuzügl. 2,50 € Versandkosten; Jahresabo $75 €$; Studenten/Ärzte in Aus- und Weiterbildung $60 €$, jeweils zuzügl. Versand (Inland $21 €$, Ausland $36 €$ ).

Bestellungen nimmt der Verlag und jede Buchhandlung entgegen. Das Abonnement verlängert sich jeweils um ein Jahr, wenn es nicht zwei Monate vor Ende des Bezugszeitraums abbestellt wurde.

\section{Abonnentenservice:}

Tel.: o 62 21/3 45-43 04; Fax: o 62 21/3 45-42 29

Gesamtherstellung: Stürtz $\mathrm{GmbH}$, AlfredNobel-Str. 33, D-97080 Würzburg

Originalien: Zur Veröffentlichung kommen nur Arbeiten, die an anderer Stelle weder angeboten noch erschienen sind. Die Autoren sind verpflichtet zu prüfen, ob Urheberrechte Dritter berührt werden. Alle CME-Manuskripte werden von unabhängigen Experten begutachtet. Eine redaktionelle Bearbeitung bleibt vorbehalten. Autoren, die mit vollem Namen genannt sind, veröffentlichen ihre Beiträge in alleiniger Verantwortung.

Copyright: Der Verlag behält sich das ausschließliche Recht der Verbreitung, Übersetzung und jeglicher Wiedergabe auch von Teilen dieser Zeitschrift durch Nachdruck, Fotokopie, Mikrofilm, EDV-Verwertung, Funk- oder Fernsehaufzeichnung vor. Jede gewerblich hergestellte oder benutzte Fotokopie verpflichtet nach $§ 54$ (2) UrHG zur Gebührenzahlung an die VG Wort, Abt. Wissenschaft, Goethestr. 49, D-80336 München, von der die Modalitäten zu erfragen sind.

Rechtseinräumung der Autoren: Mit der Einsendung eines Manuskripts zur Veröffentlichung überträgt der Verfasser dem Verlag für den Fall der Annahme das Recht, das Manuskript geändert oder unverändert, ganz oder teilweise in Pneumo-News und anderen Publikationen der Fachverlagsgruppe, in den zugehörigen Onlinediensten, in Onlinedatenbanken Dritter und, soweit vereinbart, gegen Nachhonorar in Sonderdrucken für Industriekunden zu nutzen.

Hinweis: Die in dieser Zeitschrift angegebenen Dosierungen - vor allem von Neuzulassungen - sollten in jedem Fall mit dem Beipackzettel der verwendeten Medikamente verglichen werden.

ISSN 1865-5467

\footnotetext{
Pressemeldung von AstraZeneca, 25.6.2010
} 